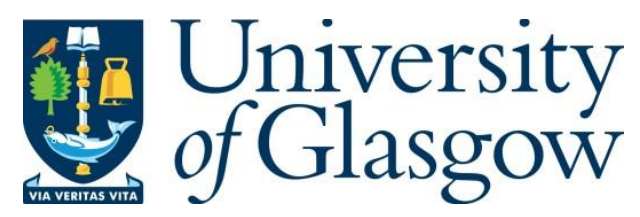

Scaffardi, M., Zhang, N., Malik, M. N., Lazzeri, E., Klitis, C., Lavery, M., Sorel, M. and Bogoni, A. (2018) Interconnection network architectures based on integrated orbital angular momentum emitters. Optics Communications, 408, pp. 63-67.

There may be differences between this version and the published version. You are advised to consult the publisher's version if you wish to cite from it.

http://eprints.gla.ac.uk/149706/

Deposited on: 19 March 2018

Enlighten - Research publications by members of the University of Glasgow http://eprints.gla.ac.uk 


\title{
Interconnection Network Architectures Based on Integrated Orbital Angular Momentum Emitters
}

\author{
Mirco Scaffardi ${ }^{1}$, Ning Zhang ${ }^{2}$, Muhammad Nouman Malik ${ }^{1,3}$, Emma Lazzeri ${ }^{3}$, Charalambos Klitis ${ }^{2}$, Martin \\ Lavery $^{2}$, Marc Sorel $^{2}$, Antonella Bogoni ${ }^{3}$ \\ (1) CNIT, Via Moruzzi 1, 56124 Pisa, Italy, e-mail: mirco.scaffardi@cnit.it \\ (2) University of Glasgow, Oakfield Avenue, Glasgow G12 8LT, UK \\ (3) Scuola Superiore Sant'Anna, Via Moruzzi 1, 56124 Pisa, Ita
}

\begin{abstract}
Novel architectures for two-layer interconnection networks based on concentric OAM emitters are presented. A scalability analysis is done in terms of devices characteristics, power budget and optical signal to noise ratio by exploiting experimentally measured parameters. The analysis shows that by exploiting optical amplifications, the proposed interconnection networks can support a number of ports higher than 100. The OAM crosstalk induced-penalty, evaluated through an experimental characterization, do not significantly affect the interconnection network performance.
\end{abstract}

Keywords: Orbital angular momentum multiplexing, optical switching, interconnection network, optical integration

\section{Introduction}

The scalability of the current electrical interconnection networks for data center connectivity is hampered by several challenging technological issues as power consumption and footprint [1,2]. An effective solution to overcome these issues is represented by optical interconnection networks based on optical switching [3]. By exploiting simultaneously different switching domains, a further enhancing of scalability and total capacity can be obtained [4]. The orbital angular momentum (OAM) of light can be exploited as further switching domain, together with the more traditional domains as e.g. wavelength, time and frequency, to increase the scalability of the optical interconnection networks. An optical beam with OAM of order $l$, where $l$ is an integer, has an azimuthal phase term $\exp (i \cdot l \cdot \theta)$, where $\theta$ is the azimuthal angle. Beams with different OAM order are orthogonal, i.e. they can propagate together ideally without crosstalk [5]. Optical beams carrying OAM can be generated by exploiting bulk components as spiral phase plates [6] or spatial light modulators [5]. Integrated devices such as microrings with super-imposed grating [7,8] circular grating couplers cascaded to star couplers [9] and hybrid 3D integrated circuits [10] have been demonstrated as a more compact alternative to the generation of light carrying OAM.

The OAM of light has been successfully exploited both in transmission and switching experiments. OAM multiplexing has been demonstrated both for free-space [5,11] and fiber transmission [12-13]. OAM switching has been demonstrated based on spatial light modulators in [14-16].

A two-layer OAM and wavelength based switch architecture has been presented in [17], based on a single and not tunable OAM emitter with multiple superimposed gratings.

Here we present three novel interconnection network architectures based on integrated concentric OAM

emitters/modulators, which can be independently tuned, thus making the network completely flexible over the two layers, i.e. OAM and wavelength. We analyze the scalability of the architectures in terms of OAM emitters/modulators general characteristics and perform a power budget and optical signal to noise ratio (OSNR) analysis. The physical parameters exploited for the analysis are experimentally measured.

\section{Two-layer interconnection network architectures}




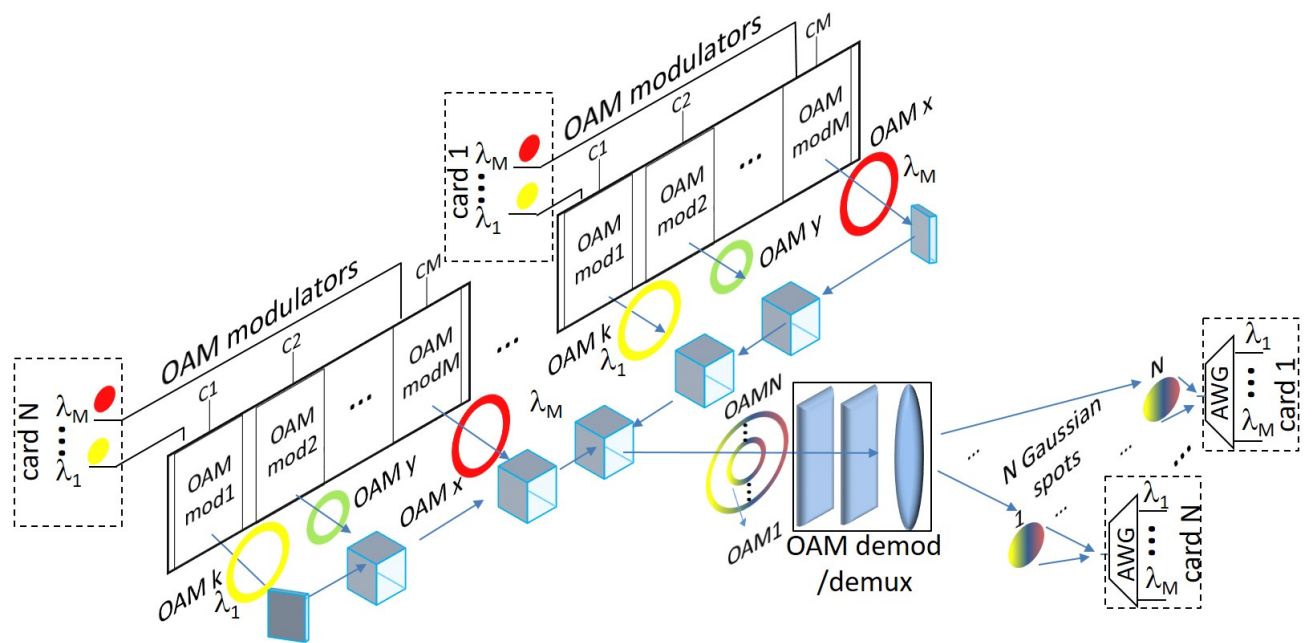

Fig. 1: Two-layer interconnection network architecture based on parallel integrated OAM emitters/modulators. A possible scheme for a two-layer interconnection network exploiting both OAM and wavelength as switching domains is shown in Fig. 1. The switch has a total number of $M \times N$ optical inputs and $M \times N$ optical outputs. The ports are logically grouped in $N$ subsets, corresponding to the number of cards of an Ethernet switch. Each set of ports represents the number of I/O ports of a card in an Ethernet switch architecture. The ports of the same subset are addressed by the wavelength domain, while the different sets of ports (i.e. cards) are addressed by the OAM domain. Each port of the OAM switch accepts a Gaussian signal, i.e. with the phase front having a Gaussian spatial distribution, at a wavelength within the allowed set of $M$ wavelengths $(\lambda 1, \ldots \lambda \mathrm{M})$. For each input port, an OAM modulator converts the signal onto an OAM mode, i.e. with a phase front with an helical spatial distribution, of order $l$ among a set of $N$ OAM modes (OAM 1, .,OAM N) depending on the targeted output ports subset. The OAM modulator can be implemented with single integrated microrings with a superimposed grating, which emits the OAM beam in a direction orthogonal with respect to the microring plane [18]. The order of the converted signal is set with a control signal (Ci) by thermal tuning [19]. All the emitted OAM modes are multiplexed by free-space multiplexing based on beam combiners. All the OAM and wavelength multiplexed beams are then sent to the OAM demodulator/demultiplexer which at the same time spatially separates the OAM modes and converts them to a wavelength-multiplexed Gaussian spot. The OAM demodulator/demultiplexer can be implemented with a passive device build with two polymetilmetacrylate (PMMA) free-space diffractive optical elements suitably patterned followed by a lens as demonstrated in [20], or with a commercial multiplane light converter [21]. Then the M wavelength are separated with an arrayed waveguide grating (AWG). This scheme allows a relatively simple technological implementation of the OAM emitters, since they can be integrated as a parallel of microrings, but introduces losses due to free-space beam combining. The maximum loss $L O$ experienced by the signals due to the free-space coupling is:

$L 0=1 / 2 \cdot\lceil(N \cdot M-1) / 2\rceil$

where $N \cdot M$ is the total number of input ports (supposed to be even). 


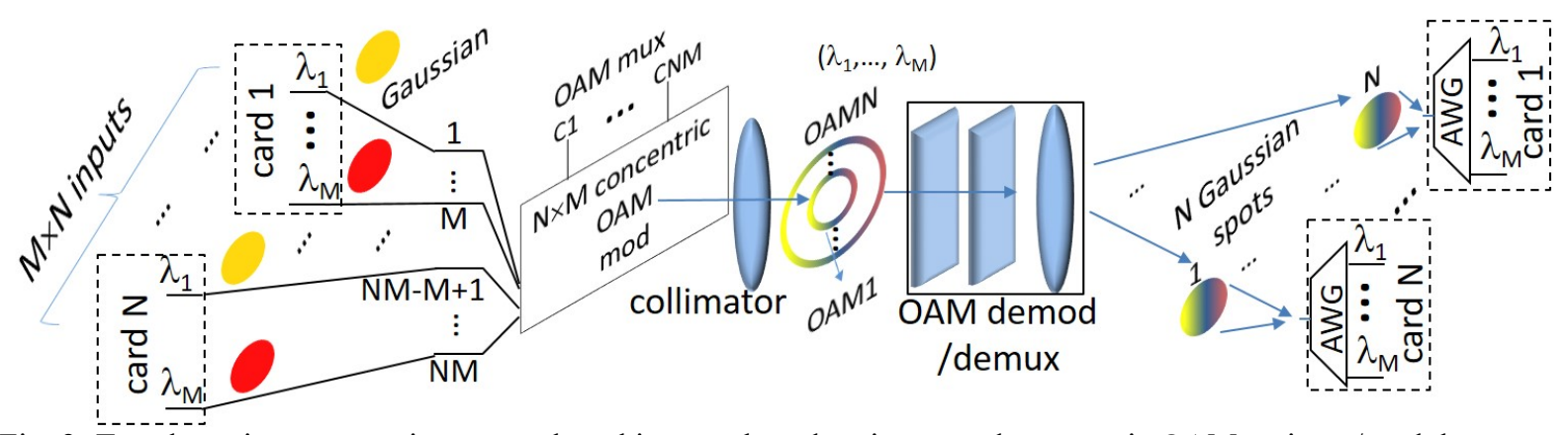

Fig. 2: Two-layer interconnection network architecture based on integrated concentric OAM emitters/modulators (OAM mux).

The number of couplers of the architecture of Fig. 1 can be reduced with an approach based on concentric OAM emitters/modulators as the one shown in Fig. 2. Here the $N \times M$ OAM emitters/modulators are concentric, i.e. the OAM signals coming out from the OAM emitters propagate coaxially, thus being spatially multiplexed. The multiplexed OAM beams are directed to the OAM demultiplexer/demodulator which spatially separates all the OAM modes of different orders and at the same time converts them to Gaussian modes, which can be propagated e.g. through a waveguide or an optical fiber. All the $M$ wavelengths are demultiplexed, i.e. spatially separated, by an AWG and directed to the corresponding output port. This scheme is very compact since the functionalities of modulation and multiplexing are implemented with a single integrated device. The number of OAM modulators that can be integrated on the same OAM mod/mux limits the number of input ports.

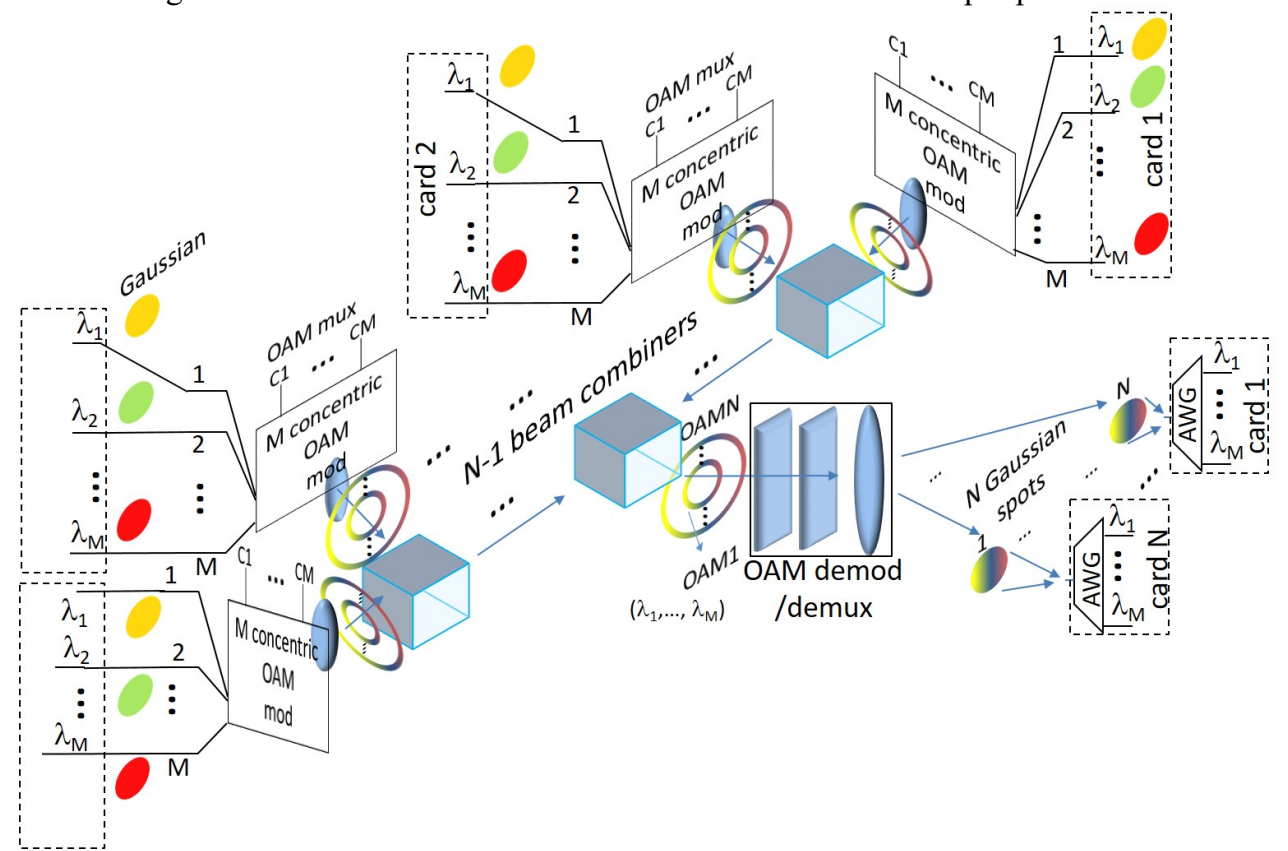

Fig. 3: Two-layer interconnection network architecture based on parallel OAM mux devices. Implementation with $N$ parallel OAM mux with $M$ concentric OAM emitter/mod per OAM mux is shown.

An hybrid approach which combines the architectures of Fig. 1 and Fig. 2 is shown in Fig. 3. This alternative architecture is based on parallel concentric OAM emitters/modulators (OAM mux). All the $M$ signals coming from each of the $N$ cards are coupled to an OAM mux implemented with $M$ concentric OAM emitters/modulators independently tunable. The emitted OAM signals are collimated and coupled with (N-1) free-space beam combiners and sent to the demodulation/demultiplexing part, which is equal to the demodulation/demultiplexing part shown in Fig. 2. This implementation reduces the number of concentric OAM emitters/modulators that need to be integrated on the same OAM mux, at the expenses of losses due to the free-space coupling. 


\section{Scalability analysis}

In order to investigate the potential of the proposed interconnection networks, a scalability analysis is developed and detailed in the following. The analysis is carried out for the two architectures shown in Fig. 2 and Fig. 3, which are more promising in terms of balancing between losses and number of ports.

In the architecture shown in Fig. 2, the total number of ports corresponds to the number of concentric OAM emitters/modulators that can be integrated on the same chip. This number is limited by the maximum allowed size (e.g. diameter) of each OAM emitter/modulator, which influences the OAM beam divergence-induced losses, and by the minimum allowed distance between two concentric emitters/modulators. The beam emitted by the concentric OAM emitters/modulators is collimated by a lens and, according to the ray optics laws, diverges depending on the diameter of the OAM emitter/modulator $(D)$ and the collimator focal length $(f)$. The OAM beam divergence should be limited in order to keep the beam spot size smaller than the aperture of the OAM demod/demux $(T)$. For a fixed maximum allowed $T$, it becomes:

$\operatorname{Dmax}<T \cdot f / L$

where $L$ is the distance between the collimator and the OAM demod/demux. Table1 shows Dmax vs. $f$ and $L$ for $T=10 \mathrm{~mm}$, which represents a safe limit for standard optical free-space devices.

\begin{tabular}{|c|c|c|c|c|c|}
\hline & \multicolumn{5}{|c|}{ Focal length $f[\mathrm{~mm}]$} \\
\hline $\begin{array}{c}\text { Distance } L \\
{[\mathrm{~mm}]}\end{array}$ & 2 & 4 & 6 & 8 & 10 \\
\hline 100 & 200 & 400 & 600 & 800 & 1000 \\
\hline 200 & 100 & 200 & 300 & 400 & 500 \\
\hline 300 & 66.6 & 133.3 & 200 & 266.6 & 333.3 \\
\hline 400 & 50 & 100 & 150 & 200 & 250 \\
\hline
\end{tabular}

Table 1: Maximum OAM emitter diameter $(\operatorname{Dmax})$ vs. collimator focal length $(f)$ and distance between collimator and OAM demux $(L)$.

The smaller is the distance between the collimator and the OAM demod/demux, the higher is the size allowed for the largest OAM emitter/modulator. Considering a collimator with high numerical aperture, e.g. NA=0.5, with $f=10 \mathrm{~mm}$, for a distance $L<200 \mathrm{~mm}, \operatorname{Dmax}>500 \mu \mathrm{m}$. The maximum number of concentric emitters in the OAM mux is:

$K=(\operatorname{Dax}-\operatorname{Dmin}) /(2 \cdot S)$

where $S$ is the minimum separation among two concentric OAM emitters and Dmin is the minimum diameter of the OAM emitter. Table 2 shows total number of ports $K=M \times N$ vs Dmax and $S$, fixing Dmin to 80 $\mu$ m [19].

\begin{tabular}{|c|c|c|c|c|c|c|}
\hline & \multicolumn{6}{|c|}{ Maximum diameter Dmax $[\mu \mathrm{m}]$} \\
\hline $\begin{array}{c}\text { Separation } \\
S[\mu \mathrm{m}]\end{array}$ & 100 & 200 & 400 & 600 & 800 & 1000 \\
\hline 2 & 5 & 30 & 80 & 130 & 180 & 230 \\
\hline 4 & 2 & 15 & 40 & 65 & 90 & 115 \\
\hline 6 & 1 & 10 & 26 & 43 & 60 & 76 \\
\hline 8 & 1 & 7 & 20 & 32 & 45 & 57 \\
\hline
\end{tabular}




\begin{tabular}{|l|l|l|l|l|l|l|}
\hline 10 & 1 & 6 & 16 & 26 & 36 & 46 \\
\hline
\end{tabular}

Table 2: Total number of ports $(N \times M)$ vs. Maximum OAM emitter diameter $($ Dmax $)$ and separation between adjacent OAM emitters $(S)$.

For $S=6 \mu \mathrm{m}, D \max =200 \mu \mathrm{m}$ is required to have 10 concentric OAM emitters. In order to have hundreds of ports, an $S<4 \mu \mathrm{m}$ is required to keep Dmax $<1 \mathrm{~mm}$. Since the minimum allowed $S$ depends on the particular technological constraints, the number of total ports can be alternatively improved by exploiting a parallel of OAM mux free-space coupled as shown in Fig. 3. The total number of ports $(N \times M)$ vs. the number of ports per OAM mux $(K)$ and the number of parallel OAM mux, is shown in Table 3. In order to have more than hundreds of ports, at least 8 OAM emitter/mod are necessary. With $K=8 \mathrm{OAM}$ emitter/mod, 128 ports can be obtained with a parallel of 16 OAM mux. In order to investigate the limitation to the scalability due to the intrinsic architectures losses, a power budget and OSNR analysis is carried out. Fig. 4 shows the scheme of the optical device chain from the transmitting to the receiving card. The chain is composed by a laser (TL), a modulator (mod), the OAM mux, the free-space combiners, the OAM demux, the arrayed waveguide grating (AWG) and the photoreceiver (PR). The power budget analysis is carried out considering the following parameters taken from commercial bulk devices: laser output power $(13 \mathrm{dBm})$ [22], laser output OSNR (55dB) [22], modulator loss (6dB) [23], OAM emitter efficiency loss (7dB), OAM demux loss $(3 \mathrm{~dB})[20]$, AWG loss (2dB) [24], photoreceiver sensitivity (-12dBm @BER 10 $\left.{ }^{-12}\right)$ [25], OSNR threshold for BER $10^{-12}=25.5 \mathrm{~dB}$ (measured from experimental characterization). The losses due to the coupling of the Gaussian beams into the concentric OAM modulators and the losses due to the coupling of the demultiplexed beam into the AWG have not been considered, since those losses do not scale with the number of ports and their value depends on the particular implementation of the switch. The received power is calculated for different numbers of parallel OAM mux, which influences the coupling losses. In the calculation, an on off keying (OOK) signal it is considered to be sent through the switch. The analysis shows that to guarantee a $\mathrm{BER}<10^{-12}$, a maximum number of 4 parallel OAM mux is allowed. In order to be able to exploit an higher number of parallel OAM mux, error correction techniques must be applied.

\begin{tabular}{|c|c|c|c|c|c|c|}
\hline & \multicolumn{5}{|c|}{ Number of parallel OAM mux } \\
\hline $\begin{array}{c}\text { Number of OAM } \\
\text { emitter/mod }(K)\end{array}$ & 2 & 4 & 8 & 12 & 16 & 20 \\
\hline 4 & 8 & 16 & 32 & 48 & 64 & 80 \\
\hline 8 & 16 & 32 & 64 & 96 & 128 & 160 \\
\hline 12 & 24 & 48 & 96 & 144 & 192 & 240 \\
\hline 16 & 32 & 64 & 128 & 192 & 256 & 320 \\
\hline 20 & 40 & 80 & 160 & 240 & 320 & 400 \\
\hline 40 & 80 & 160 & 320 & 480 & 640 & 800 \\
\hline 60 & 120 & 240 & 480 & 720 & 960 & 1200 \\
\hline
\end{tabular}

Table 3: Total number of ports $(N \times M)$ vs. the number of ports per OAM mux $(K)$ and the number of parallel OAM mux. 


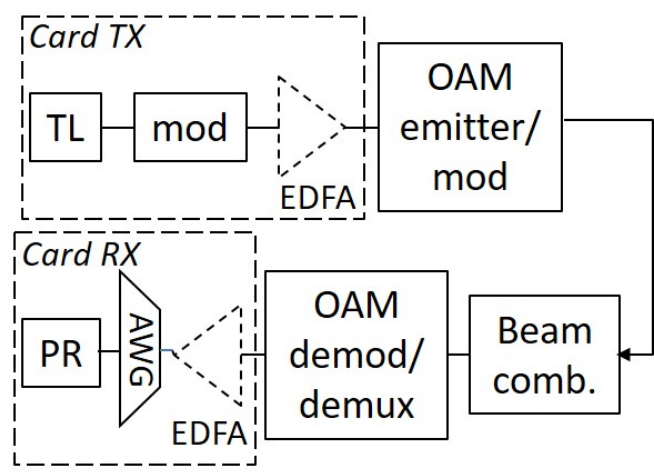

Fig. 4: Optical device chain from the transmitting to the receiving card.

Another possibility for increasing the number of switch ports is to exploit optical amplification, which allows improving the power budget. We first considered to exploit an EDFA (gain 20dB, saturation power 12dBm, noise figure $4.5 \mathrm{~dB}$ ) working as pre-amplifier before the photoreceiver, calculating the OSNR degradation due to the EDFA noise and the power budget. In this case, the number of parallel OAM mux can be increased up to 16 . If a second EDFA, working as booster, is set after the laser (gain $20 \mathrm{~dB}$, saturation power $20 \mathrm{dBm}$ ), up to 26 OAM mux can be parallelized.

The effect of the OAM crosstalk-induced penalty has been also included in the switch scalability analysis. The crosstalk is measured in an experiment where 3 consecutive OAM modes (OAM order $1=-3,-4,-5$ respectively) are generated at the same wavelength $(1556.55 \mathrm{~nm})$ with three concentric integrated OAM emitters. The measurements are performed on a $30 \mathrm{~Gb} / \mathrm{s}$ OOK signal. The BER is measured after the OAM demod/demux on the OAM channel of order $1=-3$ in two cases: only the single OAM channel of order $1=-3$ is on and all the 3 OAM channels are on. Fig. 5 shows the BER vs. received OSNR in the two cases. The crosstalk induced penalty is $<1 \mathrm{~dB}$. This low-level crosstalk does not significantly affects the power and OSNR budget analysis and do not significantly affects the performance of the proposed two-layer switch architecture. In fact the $1 \mathrm{~dB}$ OSNR penalty induced by the crosstalk do not lowers the OSNR below the threshold to obtain a BER $10^{-12}$ for a number of parallel OAM mux up to 26.

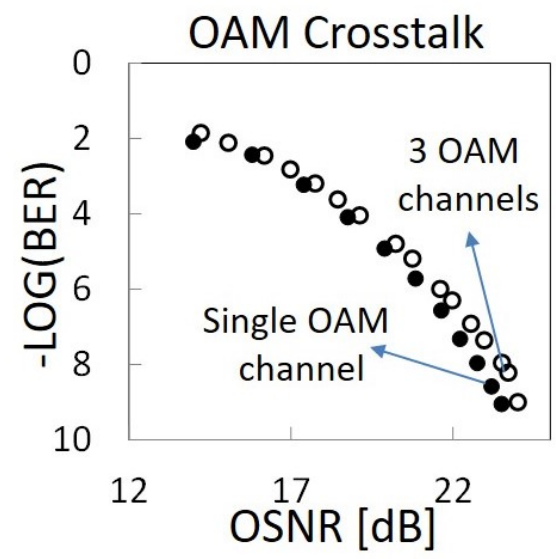

Fig. 5: BER vs OSNR for experimental OAM crosstalk evaluation.

\section{Conclusions}

Novel two-layer interconnection networks architectures are presented by exploiting OAM and wavelength as switching domains and based on concentric OAM emitter/modulators. The architectures are based on parallel OAM modulators, OAM mux with concentric OAM modulators and a parallel of OAM mux. All the architectures exploit an OAM demodulator/demultiplexer based on bulk devices.

The scalability of the architectures is analyzed in terms of OAM emitter/multiplexer characteristics (separation among the concentric emitters, diameter of the emitter, number of emitters that can be integrated on a single device) and performing a power budget and OSNR degradation analysis of the optical devices chain. In order to have an interconnection network with hundreds of ports, a parallel of 16 OAM mux with 8 concentric OAM emitter/mod per 
mux is necessary. The power budget and OSNR analysis show that by exploiting optical amplification, up to 16 parallel OAM mux can be employed, thus allowing for a number of ports higher than 100 .

The effect of the OAM crosstalk induced penalty has been also investigated. We set up an experiment where three $30 \mathrm{~Gb} / \mathrm{s}$ OOK signals at the same wavelength are mapped onto three consecutive OAM modes and the BER is measured on one of the three OAM signals after the OAM demodulator/demultiplexer in two cases: when only the signal is present and when the signals is present together with the other two interfering signals. The BER measurements indicate that the penalty introduced by the crosstalk is $<1 \mathrm{~dB}$ and do not significantly affect the scalability of the interconnection network.

\section{Acknowledgements}

This work has been funded by the Project ROAM-Revolutionizing optical fiber transmission and networking using the orbital angular momentum of light (EU H2020, contract number: 645361). The authors acknowledge support from the technical staff of the James Watt Nanofabrication Centre at Glasgow University.

\section{References}

[1] J. Humphreys, J. Scaramella, "The impact of power and cooling on data center infrastructure," Market Research Report, IDC, (2006)

[2] K. G. Brill, "The invisible crisis in the data center: the economic meltdown of Moore's law," White Paper, Uptime Institute, (2007).

[3] H. Cho, P. Kapur, K. Saraswat., "Power comparison between high-speed electrical and optical interconnects for interchip communication,’IEEE J. Lightwave Technol., 22, (2004) 2021-2033.

[4] I. Cerutti, N. Andriolli, P. G. Raponi, M. Scaffardi, O. Liboiron-Ladouceur, A. Bogoni, P. Castoldi, "Power and scalability analysis of multi-plane optical interconnection networks", IET Optoelect. 6 (2012) 192 - 200.

[5] J. Wang, J.-Y. Yang, I. M. Fazal, N. Ahmed, Y. Yan, H. Huang, Y. Ren, Y. Yue, S. Dolinar, M. Tur and A. E. Willner, "Terabit free-space data transmission employing orbital angular momentum multiplexing", Nature Photonics, 6 (2012), 488-496.

[6] X. Wei, L. Zhu, Z. Zhang, K. Wang, J. Liu, J. Wang, “Orbital Angular Momentum Multiplexing in 0.1-THz Free-Space Communication via 3D Printed Spiral Phase Plates", Proc. CLEO (2014) STu2F.2.

[7] X. Cai, J. Wang, M. J. Strain, B. Johnson-Morris, J. Zhu, M. Sorel, J. L. O’Brien, M. G. Thompson, S. Yu, "Integrated Compact Optical Vortex Beam Emitters", Science (2012) 338.

[8] M. J. Strain, X. Cai, J. Wang, J. Zhu, D. B. Phillips, L. Chen, M. Lopez-Garcia, J. L. O’Brien, M. G. Thompson, M. Sorel, S. Yu, "Fast electrical switching of orbital angular momentum modes using ultra-compact integrated vortex emitters", Nature Comm. 5 (2014) 4856.

[9] N. K. Fontaine, C. R. Doerr, L. L. Buhl, "Efficient multiplexing and demultiplexing of free-space orbital angular momentum using photonic integrated circuits", Proc. OFC, (2012) OTu1I.2.

[10] C. Quin, B. Guan, R. P. Scott, R. Proietti, N. K. Fontaine, T. Su, C. Ferarri, M. Capuzzo, F. Clemens, B. Keller, M. Earnshaw, S.J.B. Yoo "Demonstration of Orbital Angular Momentum State Conversion using Two Hybrid 3D Photonic Integrated Circuits", Proc. OFC, (2014) Th4A.1.

[11] Y. Ren, Z. Wang, P. Liao, L. Li, G. Xie, H. Huang, Z. Zhao, Y. Yan, N. Ahmed, A. Willner, M. P. J. Lavery, N. Ashrafi, S. Ashrafi, R. Bock, M. Tur, I. B. Djordjevic, M. A. Neifeld, A. E. Willner, "Experimental characterization of a $400 \mathrm{Gbit} / \mathrm{s}$ orbital angular momentum multiplexed free-space optical link over $120 \mathrm{~m}$ ", Optics Letters 41 (2016) 622-625.

[12] J. Liu, S. Li, J. Du, C. Klitis, C. Du, Q. Mo, M. Sorel, S. Yu, X. Cai, J. Wang, "Performance evaluation of analog signal transmission in an integrated optical vortex emitter to 3.6-km few-mode fiber system", Optics Letters 41 (2016) 1969-1972.

[13] J. Liu, L. Zhu, A. Wang, S. Li, S. Chen, C. Du, Q. Mo, J. Wang "All-fiber pre- and post-data exchange in kmscale fiber-based twisted lights multiplexing”, Optics Letters 41 (2016) 3896-3899.

[14] N. Ahmed, H. Huang, Y. Ren, Y. Yan, G. Xie, M. Tur, A. E. Willner, "Reconfigurable $2 \times 2$ orbital angular momentum based optical switching of 50-Gbaud QPSK channels", Optics Express 22, (2014) 756-761.

[15] A. E. Willner, L. Li, Guodong X., Yongxiong R., H. Huang, Y. Yue, N. Ahmed, M. J. Willner, A. J. Willner, Y. Yan, Z. Zhao, Z. Wang, C. Liu, M. Tur, S. Ashrafi, "Orbital-angular-momentum-based reconfigurable optical switching and routing", Photonic Research, 4 (2016) B5-B8.

[16] J. Liu, J. Wang, "Demonstration of reconfigurable joint orbital angular momentum mode and space switching", Scientific Reports 6 (2016) 37331

[17] M. Scaffardi, M. N. Malik, E. Lazzeri, G. Meloni, F. Fresi, L. Potì, N. Andriolli, I. Cerutti, C. Klitis, L. Meriggi, N. Zhang, M. Sorel, A. Bogoni., “A Silicon Microring Optical 2x2 Switch Exploiting Orbital Angular Momentum for Interconnection Networks up to 20Gbaud”, IEEE J. Light. Technol., 35 (2016) 3142-3148. 
[18] X. Cai, J. Wang, M. J. Strain, B. Johnson-Morris, J. Zhu, M. Sorel, J. L. O’Brien, M. G. Thompson, S. Yu, "Integrated Compact Optical Vortex Beam Emitters", Science 338 (2012) 363-366.

[19] M. J. Strain, X. Cai, J. Wang, J. Zhu, D. B. Phillips, L. Chen, M. Lopez-Garcia, J. L. O’Brien, M. G.

Thompson, M. Sorel, S. Yu, "Fast electrical switching of orbital angular momentum modes using ultra-compact integrated vortex emitters", Nature Comm. 5, (2014) 4856.

[20] H. Huang, G. Milione, M. P. J. Lavery, G. Xie, Y. Ren, Y. Cao, N. Ahmed, T. An Nguyen, D. A. Nolan, M.-J. Li, M. Tur, R. R. Alfano, A. E. Willner, "Mode division multiplexing using an orbital angular momentum mode sorter and MIMO-DSP over a graded-index few-mode optical fibre”, Scientific Reports 5 (2015) 14931.

[21] J.-F. Morizur, L. Nicholls, P. Jian, S. Armstrong, N. Treps, B. Hage, M. Hsu, W. Bowen, J. Janousek, H.-A.

Bachor. "Programmable unitary spatial mode manipulation", Journal of the Optical Society of America A, 27 (2010) 2524.

[22]

https://www.finisar.com/sites/default/files/downloads/finisar_s7500_cw_tunable_laser_butterfly_package_product specification.pdf

[23] Ciphotonics datasheet. $40 \mathrm{GHz}$ short-reach electroabsorption modulator.

[24]https://www.finisar.com/sites/default/files/downloads/100ghz_dwdm_48_chs_mux_demux_plug-

in module product specification_rev_d.pdf

[25] http://www.discoverysemi.com/Product_Pages/DSCR409.php 